\title{
Searching for the Holy Grail Will Need Biomarkers
}

\author{
A. Ramirez $z^{1,2,3}$ \\ 1. Department of Neurodegeneration and Geriatric Psychiatry, University of Bonn, 53127 Bonn, Germany; 2. Division of Neurogenetics and Molecular Psychiatry, \\ Department of Psychiatry and Psychotherapy, University of Cologne, Medical Faculty, 50937 Cologne, Germany; 3. German Center for Neurodegenerative Diseases (DZNE), \\ 53127 Bonn, Germany.
}

Correspondance author: Alfredo Ramirez, MD, Ph.D., Division of Neurogenetics and Molecular Psychiatry, Department of Psychiatry and Psychotherapy, University of Cologne, Kerpener Strasse 62, 50937 Cologne, Germany. Tel./Fax: +49-221-478-98041/98042, e-mail: alfredo.ramirez@uk-koeln.de.

$\mathbf{M}$ odern society benefits from the improvement of life quality and life expectancy compared to society 50 or 100 years ago (1). However, living longer is also accompanied by negative developments which include an increase in the incidence of non-communicable chronical diseases (NCDs), such as diabetes, cardiovascular diseases, neurodegenerative disorders, cancer. In fact, NCDs are becoming one of the greatest threats of our time (2). Why is that? because NCDs have a deep impact at key levels of our society, including the individuum level and the economic level, though often both levels are interconnected and influenced by each other. At the personal level, NCDs have a profound effect on the quality of life of the affected person, as well as on the person's family and on caregivers. As an example, we can consider a person who is diagnosed with dementia. For this patient begins an irreversible and unstoppable journey in the complete dependency from others and, after a given time of progression, to death, even if the patient is treated with the best disease-modifying therapies. For the patient self, this diagnose means early retirement from working life reducing consequently familial income. Given the limitation of dementia patients in their daily life activities, one or more family members will need to reduce time at work or completely take time off to care for the patient. However, increasing dependency developed by the patient will lead, sooner or later, to deep changes in the familial routine to take care of the patient that will also be associated with threatening financial problems for the complete family. Moreover, the emotional and physical drain produced while dementia progresses can be overwhelming for family and caregivers leading, sometimes, to depression and chronical stress which could last for years and incur additional cost for the family. Hence, dementia diagnosis has an enormous emotional, physical and financial burden for patients, as well as for families. From a financial perspective, the lifetime cost, in 2017, for caring for a patient with Alzheimer's disease in the USA has been estimated in $341,840 \mathrm{U} \$$ dollars (3). If we take this figure as reference, we can obtain a rough figure for the financial burden produced through the lifetime cost for the 10 million of new dementia patients diagnosed every year worldwide as reported by the world health organization in 2019 (www.who.int/news-room/fact-sheets/detail / dementia). In fact, dementia care already costs around $1 \%$ of the world's gross domestic product, and it is estimated that by the year 2050 the cost of dementia care will go up to 1.1 trillion U\$ dollars (3). Importantly, dementia is one among several NCDs that will cost the world in terms of treatment, over the next two decades (20102030), about 47 trillion U\$ dollars (4). In consequence, identifying factors and pathways involved in NCDs leading to prevention or delaying of disease onset will likely have a major impact on care cost and governmental prevention policies.

NCDs, including dementia, are multifactorial phenotypes that result from complex interactions of several factors, including genetics, epigenetics, lifestyles, and environment. Interestingly, along with the above example on dementia, several NCDs often precede dementia and are considered risk factors or first signs of ongoing dementia (5). Thus, although the clinical presentation of NCDs is different, they seem to share common pathogenic pathways. Herein, age either is the strongest risk factor or is among the most stronger risk factors for several NCDs, including diabetes, cardiovascular diseases, neurodegenerative disorders, cancer (6). In consequence, one could argue that pathological processes occurring during aging may modulate the risk of NCDs in a similar manner, and modifying these pathogenic processes might have a major impact on the prevention and treatment of dementia and NCDs. However, aging was considered for a long time an unmodifiable risk factor for NCDs. This view has changed in the last time because while chronological age cannot be stopped, research has shown that agerelated pathologic processes occurring during aging can be modified by genetic, dietary or pharmacological interventions $(7,8)$. These interventions, in turn, seem to expand lifespan in model organisms and also prevent chronical diseases. Thus, during aging, a series of biological processes lead slowly to functional decline (physical and cognitive) but in people suffering from NCDs, these processes seem to be accelerated. Which are 
the pillars (biological processes) driving aging? and How are they linked to the biology of age-related NDCs? These two questions are the main focus of the interdisciplinary field of research called "Geroscience" (8).

To increase the number of older people who "age healthily", i.e. reach older ages free of major chronic disease and disability, Geroscience will need to define one or more biomarker(s) which can inform on an agingrelated biological process and pathological development in these processes. In this issue of the Journal of Prevention of Alzheimer's Disease, Guerville et al. performed a narrative review searching in the literature for putative biomarkers of healthy aging. Herein, the authors faced the first challenge because research still lacks a clear definition of the biological processes which are tagging aging and not age-related diseases. In fact, a biomarker describing the process of aging independent of a disease might be difficult to find as medical research is focused on analyzing processes that are inherent to disease. For example, Alzheimer's disease (AD) research has been focused on the main hallmarks found in postmortem brain neuropathology, i.e. amyloid and tau. Both biomarkers might be good in reporting on the process of accumulation and aggregation of them in the brain of $\mathrm{AD}$ patients, even before symptoms appear (9). However, they are limited to a particular and specific disease process which might not necessarily correlate with aging. In fact, increased vulnerability of the brain to the initiation of neurodegenerative and nonpathological processes are thought to drive the cognitive decline observed in older individuals from the general population (10). In fact, the amyloid and tau positivity are 20-30\% less than that observed in prodromal dementia patients, independent of the age analyzed (11). Using, therefore, $\mathrm{AD}$ biomarkers as aging biomarkers to target brain aging are likely to miss key events on the aging of the brain and in general. In a similar manner using disease markers derived from different NCDs might lead to a partial or even wrong evaluation of the aging process in general. Starting from three criteria for biomarkers of aging defined by the American Federation of Aging Research, Guerville and colleagues concluded that assessing frailty, i.e. age-associated state of increased vulnerability to stressors, might better predict mortality compared to several disease-specific biomarkers which are limited to specific organs. To search the literature, the authors based their search on biomarkers reporting on the nine hallmarks of aging proposed, in 2013, by Lopez-Otin et al. (12). The main reasons for this are the fact that these hallmarks have experimental evidence linking them to modulation of aging and therefore a potential effect on healthy aging, as opposite to frailty (13).

Neither of the biomarkers reported in this review showed convincing evidence to be established as a healthy aging biomarker underscoring the fact that Geroscience still has a long way to go before healthy aging biomarkers can be used in clinical routine. Nevertheless, there are several interesting observations derived from the review that needs to be highlighted. First, the inclusion criteria used by Guerville and colleagues delivered basically only blood-based biomarkers. This also includes potential biomarkers informing on aging-relevant phenotypes operating in dementia at the systemic level. Thus, agerelated processes occurring in different organs may also influence aging and age-related pathogenic processes affecting the brain. This latter concept highlights the importance of having specific aging biomarkers reporting on the general aging pathogenic process modulating the risk of dementia, as well as other NCDs. Second, although the presented molecules cannot be included in clinical routine, a growing body of evidence supports their potential use as aging biomarkers. Third, inflammation seems to emerge as a common underlying mechanism for several of the reported molecules. For example, several lines of evidence in sirtuin-1, a marker proposed as a sensor for nutrient deregulations, showed that modulation of sirtuin- 1 activity leads to modulation of inflammation in different tissues (14). In addition, the clusterin gene (CLU) coding for the apolipoprotein-J has been involved in inflammation and immune responses by modulating the activity of complement factors, immunoglobulins, and several other signal cascades (15). Importantly, among the aging markers proposed by Guerville et al., cellular senescence involves several inflammatory mediators, beyond p16Ink4A. This makes of cellular senescence an interesting aging biomarker because it informs on several of the other hallmarks of aging. Moreover, cellular senescence has been associated with several NCDs, including dementias (16). In fact, while originally the term senescence was defined in the field of oncology, further research has established that also during neurodegenerative processes, microglia, astrocytes, and vascular unit cells become senescent (17). Herein, senescence cells showed a unique secretory profile termed the senescence-associated secretory profile (SASP), as discussed by Guerville et al. Interestingly, several of the secreted protein in the SASP include inflammatory mediators such as IL-6, TNF- $\beta$, TGF $\beta$ family ligands, IL- $1 \beta$, of which IL- $1 \beta$ is a central cytokine of senescence (16). While SASP was initially described in peripheral tissues and in association with systemic low-grade inflammation (16), SASP has been also found to be increased in cerebrospinal fluid (CSF) of AD dementia patients(18). Furthermore, in mouse models for senescence, it has been shown that modulation of the levels of SASP is associated with cognitive changes, microglia, and astrocytes activation(19, 20). Together, these data support the hypothesis of a crosstalk between peripheral and central immunosenescence related processes occurring in neurodegenerative diseases and also provide novel potential biomarkers reporting on the individual degree of senescence in the living aging brain. Furthermore, research has suggested a more general role 
of senescence-related molecules in NCDs. Interestingly, the SASP molecules and other inflammatory mediators, including proteins related to AD dementia, like TREM2 and NLRP3, have been also associated with other NCDs such as depression, stress, cardiovascular diseases, diabetes type 2, and atherosclerosis(20).

Thus, inflammation appears as a common driver for processes related to aging and NCDs, including senescence and the risk factors associated with dementia. However, each inflammatory pathway is probably modulated specifically during the life of a person. This modulation will define a unique trajectory for the cognitive decline of this person during the aging process. The shape of this trajectory will probably depend on which additional comorbidities may appear during the life of this person. Importantly, SASP molecules might probably report on the shape of these trajectories. Hence, intervention aiming to correct these inflammatory pathways might have an impact on the aging process preventing NCDs, as well as dementia. Furthermore, successful intervention on aging and senescence could in theory be followed by changes in SASP molecules. Although we are still far from the dream of aging biomarkers for clinical routine and for prevention trials, research on inflammation is delivering important evidence on the biological underpinning of SASP. Delineation of this biology and identification of the factors which modulate them will be crucial to understanding levels of SASP during aging and their variance during pathological aging. All these will be central for the development of effective treatment and improved definition of the aging group at-risk of dementia and NCDs. The review of Guerville et al. provides a very nice framework on potential hallmarks of aging and their potential biomarkers, though focusing on senescence and inflammation may deliver those so longawaited biomarkers for healthy aging and frailty.

Conflict of interest: Dr. Ramirez has no conflict of interest related with this publication.

\section{References}

1. Kirkwood TBL. Why and how are we living longer? Exp Physiol 2017;102:1067-1074.

2. Mastrangelo A, Barbas C. Chronic diseases and lifestyle biomarkers identification by metabolomics. In: Advances in Experimental Medicine and Biology Vol 965 Springer, Cham; 2017:235-263.

3. Association A. 2018 Alzheimer's disease facts and figures. Alzheimer's Dement 2018;14:367-429.

4. Chen S, Kuhn M, Prettner K, et al. The macroeconomic burden of noncommunicable diseases in the United States: Estimates and projections. PLoS One 2018;13:e0206702.

5. O'Donnell CA, Manera V, Köhler S, et al. Promoting modifiable risk factors for dementia: is there a role for general practice? Br J Gen Pract 2015;65:567-568.

6. Bales CW, Ritchie CS. Sarcopenia, weight loss, and nutritional frailty in the elderly. Annu Rev Nutr 2002;22:309-323.

7. Sierra F, Kohanski R. Geroscience and the trans-NIH Geroscience Interest Group, GSIG. GeroScience 2017;39:1-5.

8. Kennedy BK, Berger SL, Brunet A, et al. Geroscience: Linking aging to chronic disease. Cell 2014;159:709-713.

9. Jack CR, Bennett DA, Blennow K, et al. NIA-AA Research Framework: Toward a biological definition of Alzheimer's disease. Alzheimer's Dement 2018;14:535-562.

10. Boyle PA, Wilson RS, Yu L, et al. Much of late life cognitive decline is not due to common neurodegenerative pathologies. Ann Neurol 2013;74:478-489.

11. Jansen WJ, Ossenkoppele R, Knol DL, et al. Prevalence of Cerebral Amyloid Pathology in Persons Without Dementia. JAMA 2015;313:1924-38.

12. López-Otín C, Blasco MA, Partridge L, et al. The hallmarks of aging. Cell 2013;153:1194-217.

13. Michel JP, Sadana R. "Healthy Aging" Concepts and Measures. J Am Med Dir Assoc 2017;18:460-464.

14. Kitada M, Ogura Y, Monno I, et al. Sirtuins and type 2 diabetes: Role in inflammation, oxidative stress, and mitochondrial function. Front Endocrinol (Lausanne). 2019;10:187.

15. Won JC, Park C-Y, Oh SW, et al. Plasma Clusterin (ApoJ) Levels Are Associated with Adiposity and Systemic Inflammation Wang Y, ed. PLoS One 2014;9:e103351.

16. Tchkonia T, Zhu Y, Van Deursen J, et al. Cellular senescence and the senescent secretory phenotype: Therapeutic opportunities. J Clin Invest 2013;123:966972.

17. Heneka MT, McManus RM, Latz E. Inflammasome signalling in brain function and neurodegenerative disease. Nat Rev Neurosci 2018;19:610-621.

18. Tan FCC, Hutchison ER, Eitan E, et al. Are there roles for brain cell senescence in aging and neurodegenerative disorders? Biogerontology 2014;15:643-660.

19. Tha KK, Okuma Y, Miyazaki H, et al. Changes in expressions of proinflammatory cytokines IL- $1 \beta$, TNF- $\alpha$ and IL- 6 in the brain of senescence accelerated mouse (SAM) P8. Brain Res 2000;885:25-31.

20. Youm YH, Grant RW, McCabe LR, et al. Canonical Nlrp3 inflammasome links systemic low-grade inflammation to functional decline in aging. Cell Metab 2013;18:519-532. 